\title{
Long term optimal investment with regime switching: inflation, information and short sales
}

\author{
Mondher Bellalah ${ }^{1,2} \cdot$ Akeb Hakim $^{2} \cdot{\text { Kehan } \mathrm{Si}^{3}}^{3}$ Detao Zhang $^{4}(\mathrm{C})$ \\ Published online: 28 June 2020 \\ (c) Springer Science+Business Media, LLC, part of Springer Nature 2020
}

\begin{abstract}
Financial models are based on the standard assumptions of frictionless markets, complete information, no transaction costs and no taxes and borrowing and short selling without restrictions. Short-selling bans around the world after the global financial crisis and in several exchanges during the COVID 19 period, become more and more important. This paper bridges the gap by providing for the first time in the literature a model that accounting explicitly and simultaneously for inflation, information costs and short sales in the portfolio performance with regime switching. Our model can be used by portfolio managers to assess the impact of these market imperfections on portfolio decisions.
\end{abstract}

Keywords Information costs $\cdot$ Short selling costs $\cdot$ Inflation rate $\cdot$ Regime switching

JEL Classification G3 · G31 · G32 · G33

D. Zhang: This author is supported in part by Natural Science Foundation of China Grant \#11401345,11601285, 11831010, 61573217.

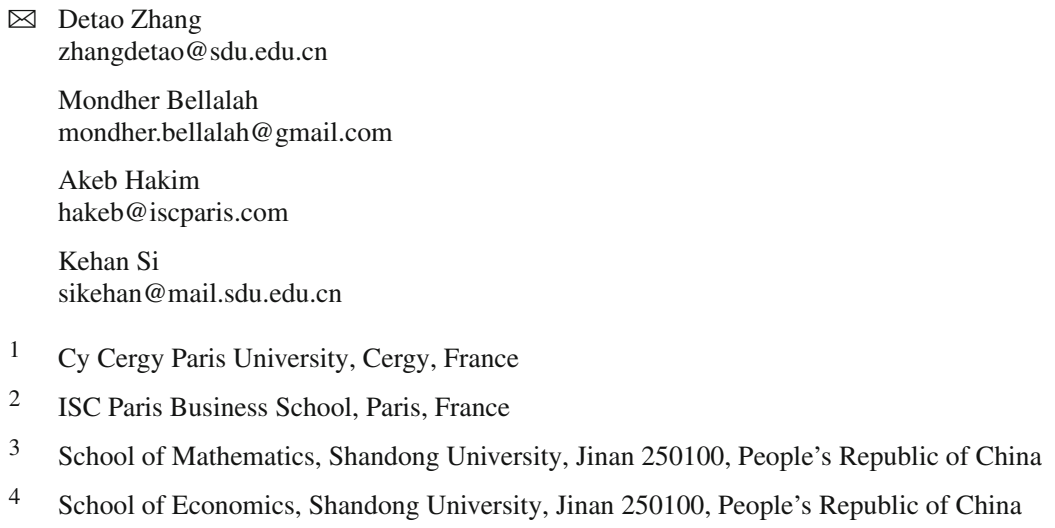




\section{Introduction}

Long term investment is very sensitive to inflation risk and to other emerging risks like COVID 19 and other emerging risks that affect portfolio decisions. How inflation can modify portfolio performance? How can we hedge portfolios against inflation in the presence of information costs and short selling? How disruptions in a given regime for unknown reasons can affect portfolio selection?

To our knowledge, no published paper has answered the question to date. Therefore, the purpose of our work is to provide clear answers to some of the above questions.

Financial models are based on the standard assumptions of frictionless markets, complete information, no transaction costs and no taxes and borrowing and short selling without restrictions. Portfolio theory refers to the work of Markowitz (1952) on portfolio selection. Investors prefer to increase their wealth and to minimize the risk linked with the potential gain. It is not possible to obtain the maximum expected return and the minimum variance.

The analysis in Merton (1987) shows that a reconciling of finance theory with empirical violations of the complete-information, perfect market model need not imply a departure from the standard paradigm. Wu et al. (1996) extend Merton's (1987) model. They propose an incomplete information capital market equilibrium with heterogeneous expectations and short sale restrictions, GCAPM. They show that the equilibrium asset returns are affected by short selling constraints and divergent beliefs. Wu et al. (1996) find that short sale restrictions mitigate the inefficiency of the market portfolio due to divergent beliefs. This is because short sales can reduce the opportunity cost of ignorance. The effect of short sales restrictions on equilibrium prices is more evident and more pronounced for smaller and less known securities, mainly because of the infrequent trading and the lack of information. The analysis increases the robustness of Merton's asset pricing model.

Short-sale constraints affect investor's use of information in financial markets. Investors who face short-sale constraints may not be able to trade based on their private information, so asset prices will not fully reflect their views. Theoretical models on short-sale constraints examine the effects of these constraints on information use by market participants. They study the implications for investment decisions and equilibrium prices. Short-sale constraints not only can affect investor's use of information in their investment decisions, but also can affect their incentives to acquire information. For example, one important type of shortsale constraint is that some investors, such as mutual fund and pension fund managers, are explicitly prohibited from short-selling. Mahdi and Wang (2013) develop a model of information acquisition and portfolio choice under short-sale constraints. The model follows the recent theoretical literature on endogenous information acquisition in financial markets and explicitly incorporates the information acquisition decision in investor's overall investment decision. We refer the reader to Nieuwerburgh and Veldkamp (2009), Van Nieuwerburgh and Veldkamp (2010), and Mackowiak and Wiederholt (2012), for models of information acquisition in financial markets. In the model by Mahdi and Wang (2013), investors take short-sale constraints into consideration in their information acquisition decisions before they acquire the information. Short sale constraints and the information acquisition decisions then jointly determine the investment decisions. In the baseline model, two assets- a risk-free asset and a risky asset- are traded in the market. Acquiring information is costly; however, doing so reduces the uncertainty that the investor is facing regarding the return of the risky asset. With short-sale constraints, the acquired information may be wasted if the investor is not allowed to sell an asset short. 
Beber and Pagano (2013) study short-selling bans around the world global financial crisis. Boehmer and Wu (2013) analyses short selling and the price discovery process. Boehmer, Jones and Zhang investigate shackling short sellers around the 2008 shorting ban. Bris et al. (2007) study the efficiency and the bear and focus on short sales and markets around the world. Cabrales et al. (2013) study entropy and the value of information for investors. Cao (1999) investigates the effect of derivative assets on information acquisition and price behavior in rational expectations equilibrium. Cao et al. (2007) study short-sale constraint, informational efficiency, and asset price bias. Massa (2002) analyses financial innovation and information and they focus on the role of derivatives when a market for information exists. Mackowiak and Wiederholt (2012) investigate information processing and limited liability

Based on the above reasons, Merton's (1987) simple model of capital market equilibrium with incomplete information has been extended and applied efficiently for the explanations of financial assets and derivatives in several contexts by several authors. Wu et al. (1996) extend Merton (1987) model to account for Incomplete-information and short sale restrictions, GCAPM. Bellalah (1999), Bellalah and Wu (2009) extend these models the model to the valuation of derivatives with information costs. We generalize the models to account also for shadow costs of short sales. This paper is organized as follows.

This paper studies how the inflation rate affect optimal portfolio choice within regime switching within information costs and short sales as described above. We solve the portfolio optimization problem of an investor who searches for the optimal investment portfolio to maximize the expected utility of real wealth using the famous Pontryagin stochastic maximum principle. Mkaouar and Prigent (2014) examine the long term investment problem, under stochastic interest and inflation rates and incompleteness. Four basic financial assets are available on the financial market: a money market account (the cash), a real consumption good, a financial stock index and a bond with constant maturity. Chou et al. (2015) solve the inter-temporal portfolio choice problems with and without interim consumption under stochastic inflation. The applications of the regime-switching model in finance have received significant attention in recent years. It modulates the system with a continuous-time finitestate Markov chain with each state representing a regime of the system or level of economic indicator, which depends on the market mode that switches among finite number states. The market mode could reflect the state of the underlying economy, the general mood of investors in the market, and other economic factors. For example, in the stock market, the up-trend volatility of a stock tends to be smaller than its down-trend volatility, therefore, it is reasonable to describe the market trends by a two-state Markov chain (see Zhang (2001) for further details). The regime-switching model has also been applied in an investmentconsumption problem in Zariphopoulou (1992). Moreover, Zhou and Yin (2003) studied a dynamic Markowitz problem for a market consisting of one bank account and multiple stocks. Maximum principle was first formulated by Pontryagin et al.s group (1962) in the 1950 s and 1960s. For the optimal control problem with regime-switching model, Donnelly (2011) studied the sufficient maximum principle. Yang et al. (2018) and Yang et al. (2019) also studied the state-dependent switching control. Using the results about BSDEs with Markov chains, Tao and $\mathrm{Wu}$ (2012) derived the maximum principle for the forward-backward regime-switching model. Recently, Wang and Wu (2015), obtained the maximum principle for forward-backward regime-switching systems involving impulse controls. Lv et al. (2016) studied the maximum principle for optimal control problem of stochastic system, which is described by an anticipated forward backward stochastic differential delayed equation and modulated by a continuous-time finite-state Markov chain.

The paper is structured as follows. In Sect. 2, we represent survey of the literature of capital market equilibrium, asset pricing and derivatives under shadow costs of incomplete 
information costs and short sales. This is the case when information is valuable and markets see a high offer on shares and other financial assets.

In Sect. 3, we present some preliminary assumptions, and form the optimal investment problem with regime-switching including stochastic inflation rate.

In Sect. 4, we obtain the optimal proportion using the Pontryain stochastic maximum principle.

In Sect. 5, we run simulations about the optimal proportion and optimal true wealth process within the general context of costly information and short sales constraints.

\section{Survey of the literature of capital market equilibrium, asset pricing and derivatives under shadow costs of incomplete information and short sales}

Financial models based on the standard assumptions of frictionless markets, no transaction costs and no taxes, and borrowing and short selling without restrictions. Merton's (1987) model based on the standard assumptions of frictionless markets, no transaction or information costs and no taxes, and borrowing and short selling without restrictions

Merton's (1987) model based on the standard assumptions of frictionless markets, no transaction costs and no taxes, and borrowing and short selling without restrictions. There are $n$ firms in the economy and $N$ investors. Investors pay information costs before they include assets in their portfolios to be informed about the specific features of the assets. Information costs are the costs of gathering and processing data, and the cost of information transmission from one party to another Information comes from the firm, stock market advisory services, brokerage houses, professional portfolio managers, etc. The model gives a general method for discounting future cash flows under uncertainty. Merton (1987) shows that the effect of incomplete information on equilibrium price is similar to applying an additional discount rate.

Wu et al. (1996) extend Merton's (1987) model to account for heterogeneous expectations and short sale restrictions. They find that short sale restrictions mitigate the inefficiency of the market portfolio due to divergent beliefs. This is because short sales can reduce the opportunity cost of ignorance. In their model, systematic risk is affected not only by the beta but also the variance of residual return and the size of the company. The model of Wu et al. (1996) parallels the model in Merton (1987) with a main difference: there are two shadow costs, $\lambda_{k}$ and $\gamma_{k}$ associated separately with the information constraint in their Eq. (9a) and short-selling constraint in their Eq. (9b). The shadow cost includes two components. The first component $\lambda_{k}$ is the product of pure information cost due to imperfect knowledge and heterogeneous expectations. The second component $\gamma_{k}$ represents the additional cost caused by the short-selling constraint. The shadow cost associated with the short-selling constraint should appear even in the case of homogeneous beliefs due to the difference in investor $j$ 's information set. In the case of divergent beliefs, the shadow cost of short sales would not be the same for all investors. Short-sale restrictions increase the likelihood that an investor will not expend the resources to be informed about a security. This tends to lower the expected payoff from acquiring the information about a security.

Wu et al. (1996) develop the following Generalized Capital Asset Pricing Model (GCAPM) to account for information costs and short sales constraints:

$$
R_{k}-R=\beta_{k}\left[R_{m}-R\right]+\left(\lambda_{k}-\gamma_{k}\right)-\beta_{k} \lambda_{m} .
$$


It can be written simply as:

$$
R_{k}=\left(R+\lambda_{k}-\gamma_{k}\right)+\beta_{k}\left[R_{m}-\lambda_{m}-R\right] .
$$

The equilibrium risk premium in GCAPM for security $k$ differs from that in the traditional CAPM. The shadow cost includes two components. The first component $\lambda_{k}$ is the product of pure information cost due to imperfect knowledge and heterogeneous expectations. The second component $\gamma_{k}$ represents the additional cost caused by the short-selling constraint. That is the expected return would be the riskless rate plus information costs less the costs of short selling. The equilibrium risk premium in GCAPM for security $k$ differs from that in the traditional CAPM. The short-sale restriction affects the shadow cost through its direct effects on portfolio weights and indirect effects on equilibrium security values. The shortsale restrictions shift the efficient set to the right and hence cause the market portfolio to be inefficient. Merton's model is a special case of the heterogeneous expectations model in GCAPM. Since $\lambda_{k}$ and $\gamma_{k}>0$, the sign of $\delta_{k}=\lambda_{k}-\gamma_{k}$ will depend on whether $\lambda_{k}$ outweighs $\gamma_{k}$.

\section{Preliminaries and problem formulation}

For sake of convenience, let us state the problem in detail below. Let $\left\{\Omega, \mathscr{F},\left\{\mathscr{F}_{t}\right\}_{0 \leq t \leq T}, P\right\}$ be a complete filtered probability space equipped with a natural filtration $\mathscr{F}_{t}$ generated by $\left\{B_{s}, \alpha_{s} ; 0 \leq s \leq t\right\}, t \in[0, T]$, where $\left\{B_{t}\right\}_{0 \leq t \leq T}$ is a $d$-dimensional standard Brownian motion defined on the space, $\left\{\alpha_{t}\right\}_{0 \leq t \leq T}$ is a finite-state Markov chain with the state space given by $I=\{1,2, \ldots, D\}$, and $T \geq 0$ is a fixed time horizon. The transition intensities are $\lambda(i, j)$ for $i \neq j$ with $\lambda(i, j)$ nonnegative and bounded. $\lambda(i, i)=-\sum_{j \in I \backslash\{i\}} \lambda(i, j)$. For $p \geq 1$, denote by $S^{p}\left(\mathbb{R}^{n}\right)$ the set of $n$-dimensional adapted processes $\left\{\varphi_{t}, 0 \leq t \leq T\right\}$ such that $\mathbb{E}\left[\sup _{0 \leq t \leq T}\left|\varphi_{t}\right|^{p}\right]<+\infty$ and denote by $H^{p}\left(\mathbb{R}^{n}\right)$ the set of $n$-dimensional adapted processes $\left\{\psi_{t}, 0 \leq t \leq T\right\}$ such that $\mathbb{E}\left[\left(\int_{0}^{T}\left|\psi_{t}\right|^{2} \mathrm{~d} t\right)^{p / 2}\right]<+\infty$.

Define $\mathscr{V}$ as the integer-valued random measure on $\left([0, T] \times I, \mathscr{B}([0, T]) \otimes \mathscr{B}_{I}\right)$ which counts the jumps $\mathscr{V}_{t}(j)$ from $\alpha$ to state $j$ between time 0 and $t$. The compensator of $\mathscr{V}_{t}(j)$ is $\mathbf{1}_{\left\{\alpha_{t} \neq j\right\}} \lambda\left(\alpha_{t}, j\right) \mathrm{d} t$, which means $\mathrm{d} \mathscr{V}_{t}(j)-\mathbf{1}_{\left\{\alpha_{t} \neq j\right\}} \lambda\left(\alpha_{t}, j\right) \mathrm{d} t:=\mathrm{d} \widetilde{\mathscr{V}}_{t}(j)$ is a martingale (compensated measure). The the canonical special semimartingale representation for $\alpha$ is given by

$$
\mathrm{d} \alpha_{t}=\sum_{j \in I} \lambda\left(\alpha_{t}, j\right)\left(j-\alpha_{t}\right) \mathrm{d} t+\sum_{j \in I}\left(j-\alpha_{t-}\right) \mathrm{d} \widetilde{\mathscr{V}}_{t}(j) .
$$

Define $n_{t}(j):=\mathbf{1}_{\left\{\alpha_{t} \neq j\right\}} \lambda\left(\alpha_{t}, j\right)$. Denote by $\mathscr{M}_{\rho}$ the set of measurable functions from $\left(I, \mathscr{B}_{I}, \rho\right)$ to $\mathbb{R}$ endowed with the topology of convergence in measure and $\left|v_{t}\right|:=$ $\sum_{j \in I}\left[v(j)^{2} n_{t}(j)\right]^{1 / 2} \in \mathbb{R}_{+} \cup\{+\infty\}$ the norm of $\mathscr{M}_{\rho}$; denote by $H_{\mathscr{V}}^{p}$ the space of $\widetilde{P}-$ measurable functions $V: \Omega \times[0, T] \times I \rightarrow \mathbb{R}$ such that $\sum_{j \in I} \mathbb{E}\left[\left(\int_{0}^{T} V_{t}(j)^{2} n_{t}(j) \mathrm{d} t\right)^{p / 2}\right]<$ $+\infty$.

After specifying the intuition of the model and the general context, we present the following model. The investment of the funds generally consists of two tradable financial assets, risky asset and risk-less asset, where for convenience, we regard the risk-less asset as risk-free one. Therefore, we assume there exists a risk-free asset which grows exponentially for $t \in[0, T]$ at the rate $r(t)$,

$$
\mathrm{d} S_{t}^{0}=r(t) S_{t}^{0} \mathrm{~d} t
$$


In the decision process for asset allocation, the information costs and short selling constraints affect the asset returns. For simplicity, we assume they are both exogenous. We assume that we invest $n$ stocks as a diversified equity portfolio whose prices satisfy the following $n$ dimensional geometric Brownian motion equation,

$$
\mathrm{d} \boldsymbol{S}_{t}=D\left(\boldsymbol{S}_{t}\right)\left[\left(\boldsymbol{\mu}\left(\boldsymbol{t}, \boldsymbol{\alpha}_{t}\right)+\lambda\left(\boldsymbol{t}, \boldsymbol{\alpha}_{t}\right)-\zeta\left(\boldsymbol{t}, \boldsymbol{\alpha}_{t}\right)\right) \mathrm{d} t+\sigma\left(\boldsymbol{t}, \boldsymbol{\alpha}_{t}\right) \mathrm{d} \boldsymbol{B}_{t}\right],
$$

where $\boldsymbol{S}_{\boldsymbol{t}}=\left(S_{t}^{1}, \ldots, S_{t}^{n}\right)^{T}$ is an $n$-vector, $\boldsymbol{B}_{\boldsymbol{t}}=\left(B_{t}^{1}, \ldots, B_{t}^{k}\right)^{T}$ is a $k$-dimensional Brownian motion, $\boldsymbol{\mu}\left(\boldsymbol{t}, \boldsymbol{\alpha}_{t}\right), \lambda\left(t, \boldsymbol{\alpha}_{t}\right), \zeta\left(t, \boldsymbol{\alpha}_{t}\right)$ are $n$-vectors, $\boldsymbol{\sigma}\left(\boldsymbol{t}, \boldsymbol{\alpha}_{\boldsymbol{t}}\right)$ is an $n \times k$ matrix and $D\left(\boldsymbol{S}_{t}\right)$ is the diagonal matrix,

$$
D\left(S_{t}\right):=\operatorname{diag}\left[S_{t}^{1}, \ldots, S_{t}^{n}\right]
$$

This means that,

$$
\mathrm{d} S_{t}^{i}=S_{t}^{i}\left[\left(\mu_{i}\left(t, \alpha_{t}\right)+\lambda_{i}\left(t, \alpha_{t}\right)-\zeta_{i}\left(t, \alpha_{t}\right)\right) \mathrm{d} t+\sigma_{i}\left(\boldsymbol{t}, \boldsymbol{\alpha}_{\boldsymbol{t}}\right) \mathrm{d} \boldsymbol{B}_{t}\right],
$$

for $\forall t \in[0, T]$ and $i=1, \ldots, n$, where $\mu_{i}\left(t, \alpha_{t}\right)$ represents the instantaneous expected rates of return of the $i$ th stock, $\lambda_{i}\left(t, \alpha_{t}\right)$ is information costs parameter to obtain information of the $i$ th stock about the market price, $\sigma_{i}\left(t, \alpha_{t}\right)$ is the $i$ th row of the instantaneous volatility matrix $\sigma\left(t, \alpha_{t}\right), \zeta_{i}\left(t, \alpha_{t}\right)$ is the cost of short selling the $i$ th securities. In fact, there also exists uncertainty in the inflation. So, we denote the rate of inflation by $I_{t}$, which is stochastic and depends on the situation of the economic cycle. As we observed recently, there are some fluctuations within the inflation rate. Like some pricing processes of the stochastic assets, we use a geometric Brownian motion (called $W_{t}$ which is not the standard Brownian motion in our space) to describe the inflation rate level at time $t$ (see also Huang and Zhang (2013), Huang et al. (2020)), that is,

$$
\mathrm{d} I_{t}=I_{t}\left[\theta(t) \mathrm{d} t+\eta(t) \mathrm{d} W_{t}\right]
$$

where the drift term $\theta(t)$ is the expected rate of inflation per unit of time, and it is defined by

$$
\theta(t)=\lim _{\delta \downarrow 0} \mathbb{E}\left(\frac{I_{t+\delta}-I_{t}}{\delta I_{t}}\right)
$$

and $\eta(t)^{2}$ is the variance of the process per unit of time defined by

$$
\eta(t)^{2}=\lim _{\delta \downarrow 0}\left[\frac{1}{\delta}\left(\frac{I_{t+\delta}-I_{t}}{I_{t}}-\delta \theta(t)\right)^{2}\right] .
$$

We assume that the trading of the investor is self-financed, i.e., there is no infusion or withdrawal of funds over $[0, T]$. We denote by $X(t)$ the amount of money of the investor at time $t$, with some initial endowment $X_{0}>0$, and denote by $\pi(t)$ the proportion of money invested in the stock, which is an $1 \times n$ matrix. The proportion of money invested in the bond is $\pi_{0}(t)$. Note that we allow short-selling so that the portfolio $\pi_{i}(\cdot)<0, i=0,1, \ldots, n$, would be allowed. Under the notation and interpretations, we have

$$
\mathrm{d} X(t)=X(t) \pi_{0}(t) \frac{\mathrm{d} S_{t}^{0}}{S_{t}^{0}}+X(t) \pi(t) D\left(S_{t}\right)^{-1} \mathrm{~d} S_{t} .
$$


We can derive that the amount of money of the investor is modeled by

$$
\left\{\begin{aligned}
\mathrm{d} X(t)= & X(t)\left[r(t)-\pi(\boldsymbol{t})\left(\boldsymbol{\mu}\left(\boldsymbol{t}, \boldsymbol{\alpha}_{t}\right)+\lambda\left(t, \boldsymbol{\alpha}_{t}\right)-\zeta\left(t, \boldsymbol{\alpha}_{t}\right)-r(t) \boldsymbol{I}\right)\right] \mathrm{d} t \\
& +X(t) \pi(t) \sigma\left(\boldsymbol{t}, \boldsymbol{\alpha}_{\boldsymbol{t}}\right) \mathrm{d} \boldsymbol{B}_{\boldsymbol{t}}, \\
X(0)= & X_{0}
\end{aligned}\right.
$$

where $\boldsymbol{I}$ is an $n \times 1$ matrix whose every components is 1 . Take the inflation rate into the consideration. Denoted by $x(t)$ the true value of assets at time $t$, which has a clear relation with $X(t)$ i.e.,

$$
x(t)=\frac{X(t)}{I_{t}} .
$$

By Itǒ's formula, we have,

$$
\mathrm{d} x(t)=\frac{\mathrm{d} X(t)}{I_{t}}-\frac{X(t)}{I_{t}^{2}} \mathrm{~d} I_{t}+\frac{X(t)}{I_{t}^{3}}\left(\mathrm{~d} I_{t}\right)^{2}-\frac{1}{I_{t}^{2}} \mathrm{~d} X(t) \mathrm{d} I_{t},
$$

without loss of generality, we can assume the initial inflation rate $I_{0}=1$, which means there is no inflation at the beginning. And combined with the Eqs. (3.4), (3.5) and (3.6), we can derive the differential equations of the true wealth process $x(t)$,

$$
\left\{\begin{aligned}
\mathrm{d} x(t)= & x(t)\left\{\left[\left(r(t)+\eta^{2}(t)-\theta(t)\right)+\pi(\boldsymbol{t})\left(\boldsymbol{\mu}\left(\boldsymbol{t}, \boldsymbol{\alpha}_{\boldsymbol{t}}\right)+\lambda\left(\boldsymbol{t}, \boldsymbol{\alpha}_{\boldsymbol{t}}\right)-\zeta\left(\boldsymbol{t}, \boldsymbol{\alpha}_{\boldsymbol{t}}\right)-r(t) \boldsymbol{I}\right.\right.\right. \\
& \left.\left.\left.-\eta(t) \boldsymbol{\sigma}\left(\boldsymbol{t}, \boldsymbol{\alpha}_{t}\right) \boldsymbol{\rho}\right)\right] \mathrm{d} t+\pi(\boldsymbol{t}) \boldsymbol{\sigma}\left(\boldsymbol{t}, \boldsymbol{\alpha}_{t}\right) \mathrm{d} \boldsymbol{B}_{t}-\eta(t) \mathrm{d} W_{t}\right\} \\
x(0)= & X_{0},
\end{aligned}\right.
$$

where $\boldsymbol{I}$ is an $n \times 1$ matrix whose every components is 1 , and $\rho$ is the $k \times 1$ correlation coefficients matrix between Brownian motions $\boldsymbol{B}_{t}$ and $W_{t}$. Each element $\rho_{j} \in \rho$ is the correlation coefficient between $B_{t}^{j}$ and $W_{t}, j=1, \ldots, k$. Let $J(t, x, \pi)$ be our cost function, and it is defined as below,

$$
J(\boldsymbol{\pi}(\cdot)):=\mathbb{E}\left[K \frac{[x(T)]^{1-R}}{1-R}\right],
$$

where $R$ is the coefficient of relative risk aversion (CRRA), $K$ is a positive constant. Our optimal portfolio problem can be stated as follows.

Problem 3.1 Maximize (3.8) subject to the wealth process (3.7) over $\pi(\cdot) \in H^{2}\left(\mathbb{R}^{n}\right)$.

At the last of this section, we summarize the assumptions on coefficients of state dynamics and cost functionals:

(A1) The coefficients of the state equations satisfy the following:

$$
\left\{\begin{array}{l}
r(\cdot), \theta(\cdot), \eta(\cdot) \in C\left(0, T ; \mathbb{R}^{n}\right), \\
\boldsymbol{\mu}(\cdot, j), \lambda(\cdot, j), \zeta(\cdot, j) \in C\left(0, T ; \mathbb{R}^{n}\right), \quad \forall j \in I, \\
\sigma_{i}(\cdot, j) \in C\left(0, T ; \mathbb{R}^{k}\right), \quad i=1,2, \cdots, n, \quad \forall j \in I .
\end{array}\right.
$$

(A2) The coefficients of the cost functionals satisfy the following:

$$
K, R>0, \quad R \neq 1 .
$$

(A3) The initial states $X_{0}>0$ is deterministic. 


\section{Optimal portfolio choice}

In this section, we want to take the advantage of the stochastic maximum principle to solve out the optimal portfolio $\pi^{*}(\boldsymbol{t})$ as described above. At first, we can define the Hamiltonian function,

$$
\begin{aligned}
H\left(t, \alpha_{t}, x(t), p(t), q(t), k(t), \boldsymbol{\pi}(\boldsymbol{t})\right): & =p(t) x(t)\left[\left(r(t)+\eta^{2}(t)-\theta(t)\right)+\boldsymbol{\pi}(\boldsymbol{t})\left(\boldsymbol{\mu}\left(\boldsymbol{t}, \boldsymbol{\alpha}_{\boldsymbol{t}}\right)\right.\right. \\
& \left.\left.+\lambda\left(\boldsymbol{t}, \boldsymbol{\alpha}_{\boldsymbol{t}}\right)-\zeta\left(\boldsymbol{t}, \boldsymbol{\alpha}_{\boldsymbol{t}}\right)-r(t) \boldsymbol{I}-\eta(t) \boldsymbol{\sigma}\left(\boldsymbol{t}, \boldsymbol{\alpha}_{\boldsymbol{t}}\right) \boldsymbol{\rho}\right)\right] \\
& +\boldsymbol{\pi}(\boldsymbol{t}) \boldsymbol{\sigma}\left(\boldsymbol{t}, \boldsymbol{\alpha}_{\boldsymbol{t}}\right) \boldsymbol{q}(\boldsymbol{t}) x(t)-\eta(t) k(t) x(t),
\end{aligned}
$$

where $\left(p(t), \boldsymbol{q}(\boldsymbol{t}), k(t), M_{t}\right)$ is the solution of the following adjoint equations:

$$
\left\{\begin{aligned}
-\mathrm{d} p(t)= & \left\{\left[\boldsymbol{\pi}(\boldsymbol{t})\left(\boldsymbol{\mu}\left(\boldsymbol{t}, \boldsymbol{\alpha}_{\boldsymbol{t}}\right)+\lambda\left(\boldsymbol{t}, \boldsymbol{\alpha}_{\boldsymbol{t}}\right)-\zeta\left(\boldsymbol{t}, \boldsymbol{\alpha}_{\boldsymbol{t}}\right)-r(t) \boldsymbol{I}-\eta(t) \boldsymbol{\sigma}\left(\boldsymbol{t}, \boldsymbol{\alpha}_{\boldsymbol{t}}\right) \boldsymbol{\rho}\right)\right.\right. \\
& \left.\left.+\left(r(t)+\eta^{2}(t)-\theta(t)\right)\right] p(t)+\boldsymbol{\pi}(\boldsymbol{t}) \boldsymbol{\sigma}\left(\boldsymbol{t}, \boldsymbol{\alpha}_{\boldsymbol{t}}\right) \boldsymbol{q}(\boldsymbol{t})-\eta(t) k(t)\right\} \mathrm{d} t \\
& -\boldsymbol{q}(\boldsymbol{t})^{T} \mathrm{~d} \boldsymbol{B}_{\boldsymbol{t}}-k(t) \mathrm{d} W_{t}-\sum_{j \in I} M_{t}(j) \mathrm{d} \mathscr{W}_{t}(j), \\
p(T)= & K(x(T))^{-R} .
\end{aligned}\right.
$$

From the reference Wang and Wu (2007), we have the following lemma,

Lemma 4.1 (Maximum Principle) Let $\boldsymbol{\pi}$ be the optimal portfoliofor the risk sensitive problem (2.1), $x(\cdot)$ be the corresponding optimal trajectory, and $(p(t), q(t), k(t), M(t))$ is the solution of adjoint $E q$. (4.2). Then for any $\pi \in H^{2}\left(\mathbb{R}^{n}\right)$, we have, a.s. a.e.,

$$
\left\langle H_{\pi}\left(t, \alpha_{t}, x(t), p(t), \boldsymbol{q}(\boldsymbol{t}), k(t), \boldsymbol{\pi}(\boldsymbol{t})\right), \boldsymbol{\pi}-\boldsymbol{\pi}(\boldsymbol{t})\right\rangle \leq 0 .
$$

From (4.3), we can derive that

$$
p(t)\left(\mu\left(t, \alpha_{t}\right)+\lambda\left(t, \alpha_{t}\right)-\zeta\left(t, \alpha_{t}\right)-r(t) I-\eta(t) \sigma\left(t, \alpha_{t}\right) \rho\right)+\sigma\left(t, \alpha_{t}\right) q(t)=0
$$

we can not directly figure out the optimal portfolio $\pi^{*}(t)$ from the formula (4.4). Because there doesn't explicitly contain $\pi(t)$ term in our cost function $J(t, x, \pi)$. To get the explicit form of optimal portfolio $\pi^{*}(t)$, the usual solving method is to use Feynman-Kac formula to derive a partial differential equation (PDE), then combine the maximum condition to obtain the desired result. However, it is difficult to obtain the explicit solution to the PDE. But if we take the advantage of the special form of the terminal condition of adjoint Eq. (4.2), we can give a direct formulation method to avoid the complicated computation steps.

Before we introduce the method, we need to get the Itǒ's formula with Markov chains. From the reference Donnelly (2011), we have the following lemma,

Lemma 4.2 (Itǒ's formula) Suppose we are given an $N$-dimensional process $X=$ $\left(X_{1}, \ldots, X_{N}\right)^{\mathrm{T}}$ satisfying for each $n=1, \ldots, N$

$$
\left\{\begin{aligned}
\mathrm{d} X_{n}(t) & =b_{n}\left(t, X(t), \alpha\left(t_{-}\right)\right) \mathrm{d} t+\sum_{m=1}^{N} \sigma_{n m}\left(t, X(t), \alpha\left(t_{-}\right)\right) \mathrm{d} W_{m}(t), \\
X_{n}(0) & =x_{0}^{(n)}, \quad \text { a.s. },
\end{aligned}\right.
$$


for some $x_{0}^{(n)} \in \mathbb{R}$, and functions $V(\cdot, \cdot, i) \in C^{1,3}\left([0, T] \times \mathbb{R}^{N}\right)$ for each $i=1, \ldots, D$. Then

$$
\begin{aligned}
V\left(t, X(t), \alpha_{t}\right)= & V(0, X(0), \alpha(0))+\int_{0}^{t} \Gamma V\left(s, X(s), \alpha\left(s_{-}\right)\right) \mathrm{d} s \\
& +\sum_{n=1}^{N} \int_{0}^{t} \frac{\partial V}{\partial x_{n}}\left(s, X(s), \alpha\left(s_{-}\right)\right) \sum_{m=1}^{N} \sigma_{n m}\left(s, X(s), \alpha\left(s_{-}\right)\right) \mathrm{d} W_{m}(s) \\
& +\sum_{j \neq i} \int_{0}^{t}(V(s, X(s), j)-V(s, X(s), i)) \mathrm{d} \mathscr{V}_{t}(j),
\end{aligned}
$$

for

$$
\begin{aligned}
\Gamma V(t, x, i):= & \frac{\partial V}{\partial t}(t, x, i)+\sum_{n=1}^{N} \frac{\partial V}{\partial x_{n}}(t, x, i) b_{n}(t, x, i) \\
& +\frac{1}{2} \sum_{n=1}^{N} \sum_{m=1}^{N} \frac{\partial^{2} V}{\partial x_{n} \partial x_{m}}(t, x, i) \sum_{l=1}^{N} \sigma_{n l}(t, x, i) \sigma_{m l}(t, x, i) \\
& +\sum_{j=1}^{D} g_{i j}(V(t, x, j)-V(t, x, i)),
\end{aligned}
$$

for all $(t, x, i) \in[0, T] \times \mathbb{R}^{N} \times I$.

Theorem 4.3 The optimal investment portfolio for problem 3.1 is given as follows

$$
\pi^{*}(t)^{T}=\left(R \sigma \sigma^{T}\left(t, \alpha_{t}\right)\right)^{-1}\left(\mu\left(t, \alpha_{t}\right)+\lambda\left(t, \alpha_{t}\right)-\zeta\left(t, \alpha_{t}\right)-r(t) I-\eta(t) \sigma\left(t, \alpha_{t}\right) \rho\right) .
$$

\section{Proof Let}

$$
p\left(t, \alpha_{t}\right)=[x(t)]^{-R} \varphi\left(t, \alpha_{t}\right)
$$

where $\varphi(\cdot, i)$ is a deterministic, differentiable function for each $i=1, \ldots, D$, which are to be found. From (4.2), $\varphi$ has terminal boundary condition

$$
\varphi(T, i)=K, \quad \forall i \in I
$$

Denote by $\widehat{b}\left(t, \alpha_{t}\right):=\left(r(t)+\eta^{2}(t)-\theta(t)\right)+\pi(t)\left(\boldsymbol{\mu}\left(\boldsymbol{t}, \boldsymbol{\alpha}_{t}\right)+\lambda\left(\boldsymbol{t}, \boldsymbol{\alpha}_{\boldsymbol{t}}\right)-\boldsymbol{\zeta}\left(\boldsymbol{t}, \boldsymbol{\alpha}_{\boldsymbol{t}}\right)-r(t) \boldsymbol{I}-\right.$ $\left.\eta(t) \sigma\left(t, \alpha_{t}\right) \rho\right), \widehat{\sigma}\left(t, \alpha_{t}\right):=\pi(t) \sigma\left(t, \alpha_{t}\right)$, and $\widehat{\eta}(t):=-\eta(t)$. Applying Itǒ's formula to $p\left(t, \alpha_{t}\right)$ defined by (4.6), we can derive that,

$$
\begin{aligned}
\mathrm{d} p\left(t, \alpha_{t}\right)= & {[x(t)]^{-R} \mathrm{~d} \varphi\left(t, \alpha_{t}\right)+\varphi\left(t, \alpha_{t}\right) \mathrm{d}[x(t)]^{-R} } \\
= & \sum_{i=1}^{D} \mathbb{1}\left[\alpha_{t_{-}}=i\right][x(t)]^{-R}\left\{-R \widehat{b}(t, i) \varphi(t, i)+\varphi_{t}(t, i)+\sum_{j \neq i} g_{i j}(\varphi(t, j)-\varphi(t, i))\right\} \mathrm{d} t \\
& +[x(t)]^{-R}\left(-R \varphi\left(t, \alpha_{t}\right) \widehat{\boldsymbol{\sigma}}\left(\boldsymbol{t}, \boldsymbol{\alpha}_{t}\right) \mathrm{d} \boldsymbol{B}_{t}-R \widehat{\eta}(t) \varphi\left(t, \alpha_{t}\right) \mathrm{d} W_{t}\right) \\
& +[x(t)]^{-R} \sum_{j \neq i}(\varphi(t, j)-\varphi(t, i)) \mathrm{d} \mathscr{V}_{t}(j),
\end{aligned}
$$


Comparing the drift term and the diffusion term of the above expression with (4.2), we have the following four equations,

$$
\begin{gathered}
\left.\varphi\left(t, \alpha_{t}\right) \widehat{b}\left(t, \alpha_{t}\right)-R \widehat{\boldsymbol{\sigma}}\left(\boldsymbol{t}, \boldsymbol{\alpha}_{\boldsymbol{t}}\right) \widehat{\boldsymbol{\sigma}}\left(\boldsymbol{t}, \boldsymbol{\alpha}_{\boldsymbol{t}}\right)^{T}-R \widehat{\eta}^{2}(t)\right) \\
=\sum_{i=1}^{D} \mathbb{1}\left[\alpha\left(t_{-}\right)=i\right]\left\{R \widehat{b}(t, i) \varphi(t, i)-\varphi_{t}(t, i)-\sum_{j \neq i} g_{i j}(\varphi(t, j)-\varphi(t, i))\right\}, \\
\boldsymbol{q}\left(\boldsymbol{t}, \boldsymbol{\alpha}_{\boldsymbol{t}}\right)=-R[x(t)]^{-R} \widehat{\boldsymbol{\sigma}}\left(\boldsymbol{t}, \boldsymbol{\alpha}_{t}\right)^{T} \varphi\left(t, \alpha_{t}\right), \\
k\left(t, \alpha_{t}\right)=-R[x(t)]^{-R} \widehat{\eta}(t) \varphi\left(t, \alpha_{t}\right), \\
\sum_{j \in I} M_{t}(j)=[x(t)]^{-R} \sum_{j \neq i}(\varphi(t, j)-\varphi(t, i)) .
\end{gathered}
$$

Substituting into (4.4) for $q(t)$ from (4.9) and using (4.6) to replace $p(t)$, we get

$$
\pi^{*}(t)^{T}=\left(R \sigma \sigma^{T}\left(t, \alpha_{t}\right)\right)^{-1}\left(\mu\left(t, \alpha_{t}\right)+\lambda\left(t, \alpha_{t}\right)-\zeta\left(t, \alpha_{t}\right)-r(t) I-\eta(t) \sigma\left(t, \alpha_{t}\right) \rho\right) .
$$

Therefore, to complete the proof, it remains to find $\varphi$, i.e. to solve out the (4.8). We obtain the equation

$$
\left((R-1) P(t)+R \eta^{2}(t)+\boldsymbol{Q}(\boldsymbol{t})^{T}\left(\boldsymbol{\sigma} \boldsymbol{\sigma}^{T}\right)^{-1} \boldsymbol{Q}(\boldsymbol{t})\right) \varphi(t, i)-\varphi_{t}(t, i)-\sum_{j \neq i} g_{i j}(\varphi(t, j)-\varphi(t, i))=0,
$$

where $P(t):=r(t)+\eta^{2}(t)-\theta(t)$ and $\boldsymbol{Q}(\boldsymbol{t}):=\boldsymbol{\mu}(\boldsymbol{t}, \boldsymbol{i})+\lambda(\boldsymbol{t}, \boldsymbol{i})-\boldsymbol{\zeta}(\boldsymbol{t}, \boldsymbol{i})-r(t) \boldsymbol{I}-$ $\eta(t) \sigma(t, i) \rho$, with the terminal boundary condition given by (4.7). Consider the process

$$
\tilde{\varphi}\left(t, \alpha_{t}\right):=K \mathbb{E}\left[\exp \left\{\int_{t}^{T} A(s) \mathrm{d} s\right\} \mid \alpha_{t}\right],
$$

where $A(s):=(R-1) P(t)+R \eta^{2}(t)+\boldsymbol{Q}(\boldsymbol{t})^{T}\left(\boldsymbol{\sigma} \sigma^{T}\right)^{-1} \boldsymbol{Q}(\boldsymbol{t})$. We aim to show that $\varphi=\tilde{\varphi}$. It is helpful to define at this point the following martingale:

$$
R(t):=\mathbb{E}\left[\exp \left\{\int_{t}^{T} A(s) \mathrm{d} s\right\} \mid \mathscr{F}_{t}^{\alpha}\right],
$$

where $\mathscr{F}_{t}^{\alpha}:=\sigma\left\{\alpha_{\tau}, \tau \in[0, t]\right\} \vee \mathscr{N}(P)$ is the filtration generated by the Markov chain. From the $\mathscr{F}_{t}^{\alpha}$-martingale representation theorem, there exists $\mathscr{F}_{t}^{\alpha}$-previsible, square-integrable process $v^{R}(t)$ such that

$$
R(t)=R(0)+\sum_{j \neq i} \int_{0}^{t} v_{i j}^{R}(\tau) \mathrm{d} \mathscr{V}_{\tau}(j)
$$

By the positivity of $R(t)$, we can define the process $\widehat{v}_{i j}^{R}(t):=v_{i j}^{R}(t) R^{-1}\left(t_{-}\right)$so that

$$
R(t)=R(0)+\sum_{j \neq i} \int_{0}^{t} R\left(\tau_{-}\right) \widehat{v}_{i j}^{R}(\tau) \mathrm{d} \mathscr{V}_{\tau}(j) .
$$

From (4.13) and the definition of $R(t)$ in (4.14), we have the relationship

$$
R(t)=\frac{1}{K} \widetilde{\varphi}\left(t, \alpha_{t}\right) \exp \left\{\int_{t}^{T} A(s) \mathrm{d} s\right\}, \quad \forall t \in[0, T] .
$$




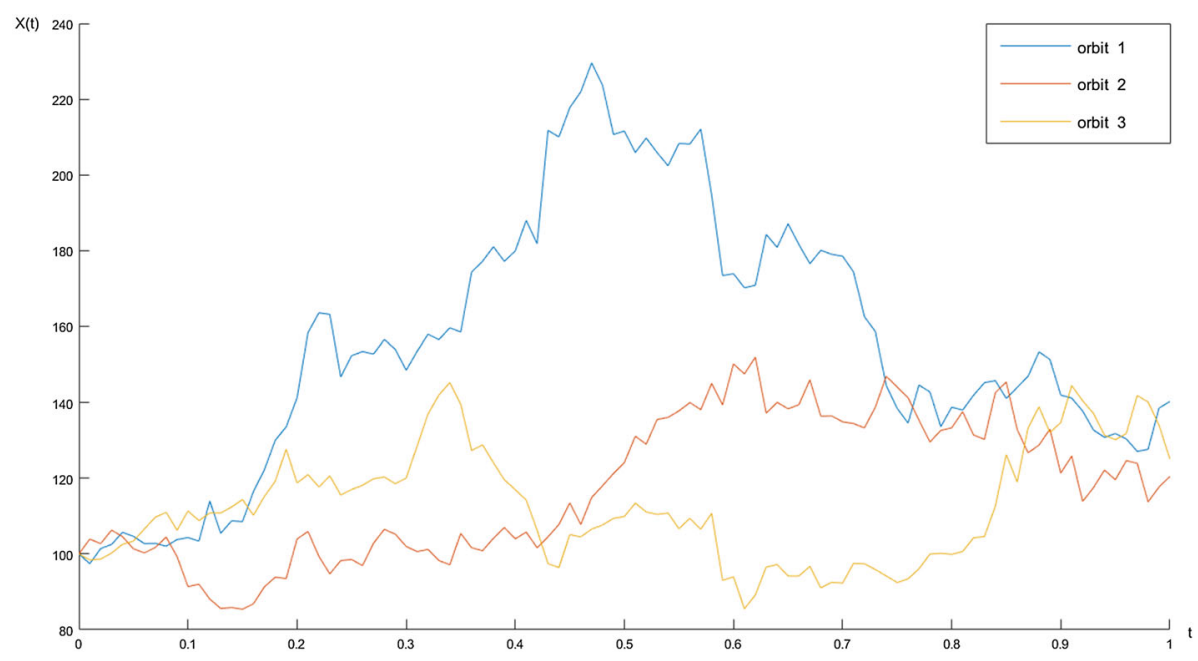

Fig. 1 Graph of $x^{*}(t)$ respect with $t$

Using the Itǒ's formula expansion of $\widetilde{\varphi}\left(t, \alpha_{t}\right)$, we apply integration-by-parts to expand the right-hand side of the above equation and comparing it with the martingale representation of $R(t)$ given by (4.15), we find that $\tilde{\varphi}$ satisfies (4.12) with $\varphi=\tilde{\varphi}$. We conclude that $\varphi=\tilde{\varphi}$.

Remark 4.4 We must point out that the optimal investment proportion $\pi^{*}$ defined by (4.5) clearly depends on the Arrow-Pratt index of risk aversion of the investor $R . \pi^{*}$ is negative correlated with the interest rate $r(t)$, the volatilities for the stock and inflation rate $\sigma\left(t, \alpha_{t}\right)$, and $\eta_{t} . \pi^{*}$ will be positive correlated with the instantaneous expected rate of return $\mu\left(t, \alpha_{t}\right)$, and the relative coefficient $\rho$ of the random interference source between the price of stock and the inflation rate. These phenomena coincide with our intuition and this confirms our results. Our results are important and can help portfolio managers in portfolio selection.

\section{Numerical simulation}

In this section, we do some simulation about the optimal portfolio choice and the optimal wealth process. For simplicity, we consider that there are only two stocks traded in the market and freeze the coefficients be constants. By the way, for the same role of the $\mu, \lambda$, and $\zeta$, we only consider one of them. Set $D=\left[i_{1}, i_{2}\right], \mu_{1}\left(i_{1}\right)=10 \%, \mu_{1}\left(i_{2}\right)=15 \%, \mu_{2}\left(i_{1}\right)=12 \%$, $\mu_{2}\left(i_{2}\right)=18 \%, \sigma_{1}\left(i_{1}\right)=[0.4,0], \sigma_{1}\left(i_{2}\right)=[0.6,0], \sigma_{2}\left(i_{1}\right)=[0,0.5], \sigma_{2}\left(i_{2}\right)=[0,0.8]$, $\theta(t)=0.05, \eta(t)=0.1, \rho_{1}=0.2, \rho_{2}=0.4, r(t)=3 \%, R=0.5, K=1, X_{0}=100$, and the intensity of the transition of the Markov chain $\alpha(t)$ is $Q=\left(\begin{array}{cc}-5 & 5 \\ 6 & -6\end{array}\right)$. Because we allow the operation of short-selling, we can accept the optimal portfolio choice $\pi$, which has the situation $\sum_{i=1}^{n} \pi_{i} \geq 1$ or $\sum_{i=1}^{n} \pi_{i} \leq 1$. Here, in fact, the states $i_{1}, i_{2}$ are denoted by the bear market and the bull market. It helps a lot when we analyse the results.

Figure 1 shows three orbits of the optimal wealth process path. It is clearly shown in the graph that every turning point of the orbits coincides, even if their orientations may differ. We also illustrate part of our results regarding optimal portfolio choice in the presence of information costs and short selling constraints in Fig. 2. he following Fig. 2 shows the graph of the optimal portfolio choice respected with $t$. It is easy to find that we will allocate more 


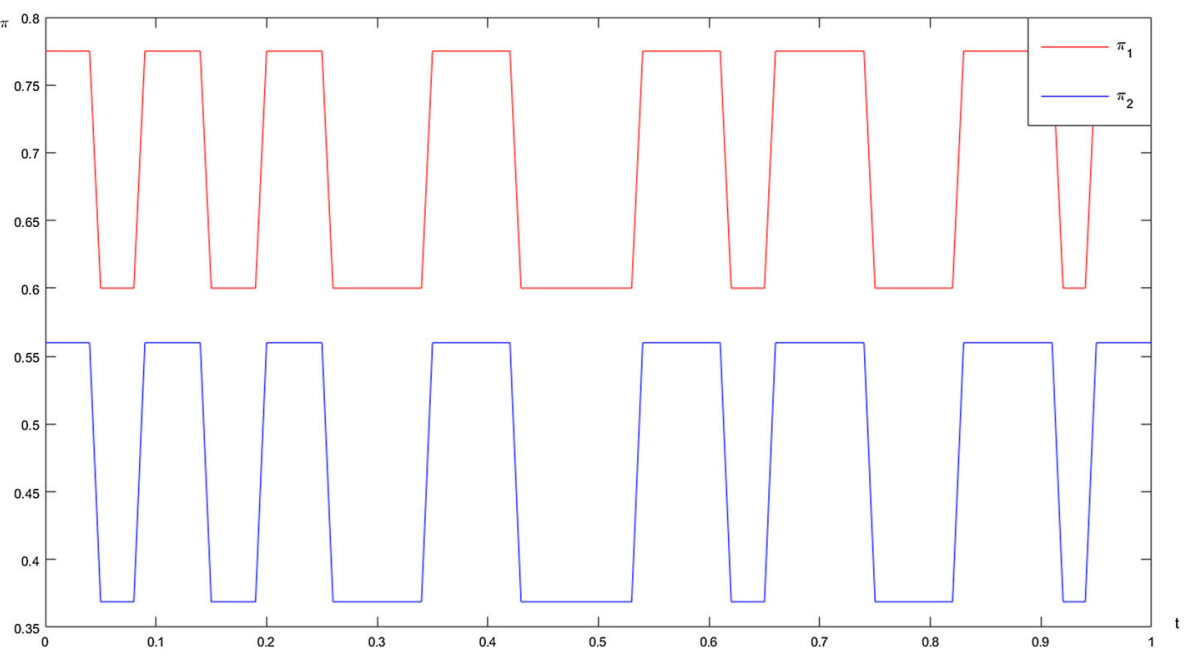

Fig. 2 Graph of $\pi^{*}$ respect with $t$

money into the stocks market in the bull market, and less money in bear markets. The actual period of COVID 19 illustrates a significant reduction in allocated investments throughout the world. The model illustrates how to switch from the market according to the periods. Our results corroborates the intuition.

\section{Conclusion}

Financial models based on the standard assumptions complete information, absence of transaction costs and taxes, borrowing, lending at the same rate, and selling without restrictions. The effects of incomplete information and short selling constraints are very important in portfolio selection. They are valuable in portfolio decisions during periods with high volatility such as the COVID 19 period in 2020.

Merton's (1987) develops a simple model of capital market equilibrium with incomplete information. Wu et al. (1996) extend Merton's (1987) model by proposing an incomplete information capital market-equilibrium with heterogeneous expectations and short sale restrictions, GCAPM. The shadow costs include two components. The first component is the product of pure information cost due to imperfect knowledge and heterogeneous expectations. The second component represents the additional cost caused by the short-selling constraint. Short-selling bans around the world after the global financial crisis become more and more important. Mahdi and Wang (2013) develop a model of information acquisition and portfolio choice under short-sale constraints. Bellalah (1999) and Bellalah and Wu (2009) include information costs the valuation of assets and derivatives. However, there is no published work to our knowledge that accounts simultaneously for inflation, information costs, short sales constraints in the market for portfolio performance with switching regime. Investors can switch regime also in periods with imbalances in portfolio such as COVID 19 period. This is the first study devoted to the performance of a portfolio that accounts simultaneously for information costs and short sales constraints. 
In this paper, we study the optimization problem with regime switching, inflation, information costs and short sales. Using the Pontryagin maximum principle, we obtain the optimal investment proportion within inflation, information costs and short sales. Some numerical simulations have been done in Sect. 5 to further support our observations. Our results are very useful and valuable for portfolio and fund managers who have to take portfolio decisions in difficult times. Our model can be tested on some empirical data.

\section{References}

Beber, A., \& Pagano, M. (2013). Short-selling bans around the world: Evidence from the 2007-2009 crisis. Journal of Finance, 68(1), 343-381.

Bellalah, M. (1999). The valuation of futures and commodity options with information costs. Journal of Futures Markets: Futures, Options, and Other Derivative Products, 19(6), 645-664.

Bellalah, M., \& Wu, Z. (2009). A simple model of corporate international investment under incomplete information and taxes. Annals of Operations Research, 165, 123-143.

Boehmer, E., Jones, C.M., \& Zhang, X. Shackling short sellers: The 2008 shorting ban, Review of Financial Studies.

Boehmer, E., \& Wu, J. (2013). Short selling and the price discovery process. Review of Financial Studies, $26(2), 287-322$.

Bris, A., Goetzmann, W. N., \& Zhu, N. (2007). Efficiency and the bear: Short sales and markets around the world. Journal of Finance, 62(3), 1029-1079.

Cabrales, A., Gossner, O., \& Serrano, R. (2013). Entropy and the value of information for investors. American Economic Review, 103(1), 360-377.

Cao, H. H., Zhang, H. H., \& Zhou, X. (2007). Short-sale constraint, informational efficiency, and asset price bias. University of Texas-Dallas.

Cao, H. H. (1999). The effect of derivative assets on information acquisition and price behavior in a rational expectations equilibrium. Review of Financial Studies, 12(1), 131-163.

Chou, Y., Han, N., \& Hung, M. (2015). Optimal portfolio consumption choice under stochastic inflation with nominal and indexed bonds. Applied Stochastic Models in Business and Industry, 27(6), 691-706.

Chunchi, W., Li, Q., \& John Wei, K. C. (1996). Incomplete information capital market equilibrium with heterogeneous expectations and short sale restrictions. Review of Quantitative Finance and Accounting, 7, 119-136.

Donnelly, C. (2011). Sufficient stochastic maximum principle in a regime-switching diffusion model. Applied Mathematics and Optimization, 64(2), 155-169.

Huang, Z., Wang, H., \& Wu, Z. (2020). A kind of optimal investment problem under inflation and uncertain time horizon. Applied Mathematics and Computation, 375, 125084.

Huang Z., Zhang D., (2013). Optimal portfolio of corporate investment and consumption problem under market closure: Inflation case. Mathematical Problems in Engineering, 715869.

Lv, S., Tao, R., \& Wu, Z. (2016). Maximum principle for optimal control of anticipated forward backward stochastic differential delayed systems with regime switching. Optimal Control Applications and Methods, 37, 154-175.

Mackowiak, B., \& Wiederholt, M. (2012). Information processing and limited liability. American Economic Review, 102(3), 30-34.

Mahdi, Nezafat, \& Wang, Qinghai. (2013). Short-sale Constraints, information acquisition, and asset prices, August, 2013, Scheller College of Business, Georgia Institute of Technology, Atlanta, GA 30308.

Markowitz, H. M. (1952). Portfolio selection. Journal of Finance, 7(1), 77-91.

Massa, M. (2002). Financial innovation and information: The role of derivatives when a market for information exists. Review of Financial Studies, 15(3), 927-957.

Merton, R. C. (1987). A simple model of capital market equilibrium with incomplete information. Journal of Finance, 42, 483-510.

Mkaouar, F., \& Prigent, J. L. (2014). Long term investment with stochastic interest and inflation rates: Incompleteness and compensating variation, Working paper.

Nieuwerburgh, V. S., \& Veldkamp, L. (2009). Information immobility and the home bias puzzle. Journal of Finance, 64(3), 1187-1215.

Pontryagin, L., Boltyanskti, V., Gamkrelidze, R., \& Mischenko, E. (1962). The mathematical theory of optimal control processes. New York: Wiley. 
Tao, R., \& Wu, Z. (2012). Maximum principle for optimal control problems of forward-backward regimeswitching system and applications. Systems and Control Letters., 61(9), 911-917.

Van Nieuwerburgh, S., \& Veldkamp, L. (2010). Information acquisition and under-diversification. Review of Economic Studies, 77(2), 779-805.

Wang, S., \& Wu, Z. (2015). Maximum principle for optimal control problems of forward-backward regimeswitching systems involving impulse controls. Mathematical Problems in Engineering,.

Wang, G., \& Wu, Z. (2007). Stochastic maximun principle for a kind of risk-sensitive optimal control problem and application to portfolio choice. Acta Automatica Sinica, 33(10), 1043-1047.

Yang, D., Li, X., \& Qiu, J. (2019). Output tracking control of delayed switched systems via state-dependent switching and dynamic output feedback. Nonlinear Analysis: Hybrid Systems, 32, 294-305.

Yang, D., Li, X., Shen, J., \& Zhou, Z. (2018). State-dependent switching control of delayed switched systems with stable and unstable modes. Mathematical Methods in the Applied Sciences, 41(16), 6968-6983.

Zariphopoulou, T. (1992). Investment-consumption models with transaction costs and Markov-chain parameters. SIAM Journal on Control and Optimization, 30, 613-636.

Zhang, Q. (2001). Stock trading: An optimal selling rule. SIAM Journal on Control and Optimization, 40, 64-87.

Zhou, X., \& Yin, G. (2003). Markowitz's mean-variance portfolio selection with regime switching: A continuous-time model. SIAM Journal on Control and Optimization, 42, 1466-1482.

Publisher's Note Springer Nature remains neutral with regard to jurisdictional claims in published maps and institutional affiliations. 